Correo: innova@uide.edu.ec

\title{
Club de Periodismo, modelo de práctica educativa para fortalecer la escritura periodística
}

\section{Journalism Club, model of educational practice to strengthen journalistic writing}

Lcda. Dennys Jordán Correa, Mgs.

Universidad Internacional del Ecuador, Ecuador

Autor para correspondencia: dejordanco@ uide.edu.ec

Fecha de recepción: 10 de Junio de 2016 - Fecha de aceptación: 28 de Junio de 2016

\section{Resumen}

La redacción periodística experimenta la necesidad de fortalecerse como requisito de las habilidades básicas del periodismo; no obstante, el dominio de la competencia discursiva escrita no siempre es parte del perfil de egreso de los profesionales. Las Facultades de Comunicación cumplen su función de enseñar pero ésta también involucra el desarrollo de estrategias de educación prácticas que favorezcan la práctica periodística; de esta manera surge el Club de Periodismo, una iniciativa que permite que los futuros profesionales fortalecer la escritura como herramienta básica de su profesión.

Palabras claves: producción; audiovisual; comunicación; teoría; educación

\begin{abstract}
Journalistic writing feels the need to be strengthened as a requirement of the basic skills of journalism; however, the domain of discourse competence is not always written part of the graduate profile of professionals. Communication Faculties fulfill their role of teaching but it also involves the development of practical education strategies that promote journalistic practice; thus the Journalism Club, an initiative that allows future professionals to strengthen writing as a basic tool of their profession arises.
\end{abstract}

Key words: production; audiovisual; communication; theory; education 


\section{Introducción}

A pesar de que la prensa escrita pierde lectores cada año, aún es el medio que homogeniza los noticieros de televisión, radio y portales web; por eso, el contenido de las portadas de papel está presente en los informativos de radio, televisión y redes sociales, así lo explicaba el exvicedirector de diario El Mundo de España, quien argumentaba que el gran valor de los periódicos radica en que "todavía son los que marcan la agenda política" y esto hace que mantenga recelo ante el pronóstico de su desaparición o de su limitación a un sitio web (GarcíaAbadillo, 2009).

La estructura ordenada, la información detallada, amplia, jerarquizada y la organización que presenta el contenido impreso mantienen el interés de los lectores, quienes prefieren este medio para ampliar el conocimiento y conocer las múltiples fuentes de la información. El periódico es, entonces, el medio principal y más directo para la difusión de noticias; y, por eso es el referente de los demás. Esta rigurosidad también está presente en la construcción del conocimiento porque la redacción periodística experimenta la necesidad de fortalecerse como requisito de las habilidades básicas de la profesión; no obstante, el dominio de la competencia discursiva escrita no siempre es parte del perfil de egreso de los profesionales.

Los comunicadores requieren interactuar en diversos espacios socioculturales. De allí la necesidad de generar iniciativas que fomenten la práctica comunicativa y, sobre todo, de la escritura, que requiere de experiencias continuas para desarrollar las habilidades cognitivas, socioculturales y lingüísticas. La Organización de la Naciones Unidas para la Educación, la Ciencia y la Cultura propuso que las instituciones de educación superior facilitaran "la adquisición de conocimientos prácticos, competencias y aptitudes para la comunicación y el análisis creativo y crítico" (Unesco, 1998) por medio de prácticas pedagógicas innovadoras.

Pero ¿en la actualidad, los periodistas en proceso están fortaleciendo y desarrollando esta competencia? Precisamente, José Hernández, en su libro Periodismo Oficio Imposible, da cuenta de que las habilidades que adquieren los periodistas en las universidades son bastante limitadas en relación a las demandas de los medios impresos. "Periodistas que creen saber de todo, pero que en realidad no escriben bien de nada; todo porque el formato del pensum académico se enmarca en forma de periodistas todólogos y eso también lo hacen con falencias" (Hernández, 2004).

Cuando se refiere a práctica, el término es vital para los periodistas porque es la única manera de esclarecer los conceptos aprendidos en las aulas. Que diferencien información de opinión es un verdadero reto para todos los profesionales del medio, así lo manifiesta el co-autor de la Ley de Comunicación, Romel Jurado. "El que informa afirma un dato, con certeza y seguridad, mientras quien opina lo que expresa, lo hace sin afirmar o negar alguna información" (Jurado, 2015). Lastimosamente en Ecuador se confunde mucho y personas no preparadas que están en los grandes medios de comunicación, siguen mezclando la opinión con la información.

Tener claridad en el punto de vista permite armar una historia. Guillermo Lizarzaburu, editor general de Diario Expreso asevera que uno de los inconvenientes de los futuros profesionales de la comunicación son las pocas herramientas de trabajo que poseen. Capacidad 
de síntesis, análisis, iniciativas para generar temas nuevos, estructuración de datos a investigar, manejo de fuentes y redacción férrea son las principales falencias que evidencia en los pasantes que van a este medio impreso.

Está convencido de que la única manera de erradicar estos problemas es que la universidad cuente con la contratación de una planta docente que trabaje en medios o haya ejercido en los mismos. "La experiencia profesional hará que el conocimiento no se vuelva una simple formulación de conceptos enciclopédicos sino que lleve a la par con la práctica, con la formulación del ejercicio profesional; de esta manera se fortalecerá la redacción en todas sus facetas porque ésta es la herramienta básica del periodista y verificar, constantemente, esa capacidad de estar informados porque es la única manera de hacer juicios más sólidos y un trabajo más verídico" (Lizarzaburo, 2015).

La Organización de las Naciones Unidas para la Educación, la Ciencia y la Cultura, (Unesco, 1998) determinó que la gestión y el financiamiento de la enseñanza superior exige capacidades y estrategias de planificación y análisis entre los establecimientos académicos y organizaciones nacionales para asegurar una gestión adecuada y recursos eficaces; es decir, esto motivó la re conceptualización de la educación, asumiéndola como formadora y, sobre todo, integral.

Esto es evidente en el nuevo siglo, en donde el desarrollo de la ciencia y la técnica es prolijo y, por ende, ha desencadenado la fusión de paradigmas del conocimiento y las tendencias pedagógicas contemporáneas. En Periodismo, estas ideas pedagógicas deberían combinar la práctica como parte del aprendizaje, dejando atrás la formación netamente literaria.

Philip Zurita, Jefe del departamento de Talento Humano de diario El Universo, indica que una prueba de conocimientos generales y una de redacción se convierten en las bases para los aspirantes a pasantes en el medio. Generalmente, casi todos los estudiantes pasaban, con dificultad, la primera herramienta; pero, en la segunda, evidenciaban los problemas de ortografía y sintaxis. Con su experiencia, propone forjar una "escuela práctica" porque, asegura, que las falencias de los casi profesionales se superará cuando adquieran más hábitos lectores porque esto es una forma de educarse culturalmente y autocorregir la ortografía.

A los nuevos periodistas les hace falta mucha práctica y, para eso, no es necesario depender de un medio; también se puede crear sus propios espacios como blogs, páginas web, periódicos, revistas que cuenten con la participación de los propios estudiantes (Zurita, 2014). Hay que fortalecer la redacción porque no se están formando de manera integral; además, se debe propiciar el trabajo de campo, porque las noticias están fuera del aula de clases; entonces, hay que aprender a informar y para esto se debe estar siempre informado.

Según investigaciones de Fernando Villalobos, Mary Alejandra Montiel, Katia Muñoz Vásquez, Sergio Celedón Díaz, miembros del Centro Internacional de Estudios Superiores de Comunicación para América Latina, Ciespal, la nueva generación de periodistas enfrenta el reto del manejo de la comunicación digital aparte de los medios tradicionales; pero, independientemente del medio donde se desenvuelvan, se necesitara las bases primordiales del periodismo: responsabilidad social, investigación, buena redacción, elocuencia y sintaxis, 
dominar los géneros periodísticos e incluso consideran oportuno la ramificación de éstos como especialidades de la carrera. Otra vez se resalta la necesidad de escribir bien como herramienta práctica del oficio.

Redactar es base; pero cómo hacerlo si además de no poseer las técnicas suficientes carece de contenido, es decir, en la actualidad, los profesionales no tienen conocimientos sociales y esto lo reafirma Juan Carlos Mestanza, ex Coordinador Regional de Diario El Comercio, quien señala cuán pertinente es ampliar los conocimientos de cultura general y el dominio de procesos de redacción periodística, de géneros periodísticos. "Indistintamente del medio (prensa, Tv o radio) debe tener muy claro los estilos y géneros periodísticos. Saber redactar una noticia es primordial. Para reforzar la formación periodística es necesario hacerse la pregunta ¿tengo vocación? Para trazar con mayor ahínco un camino de constante preparación intelectual y buscar alternativas para prepararse por su cuenta".

La práctica es requisito indispensable de esta carrera y la investigación es la esencia del verdadero periodismo y ésta permite participar, colaborar y transformar la realidad. Hoy, se plantea un nuevo desafío: lograr una correspondencia entre los fines de la institución y la exigencia del entorno- contexto para lograr que la academia adquiera una función de pertinencia, además de la propia función formadora.

Ese cambio es el que propone Fabricio Montesdeoca, editor de Diario Metro, quien señala que "es indispensable cambiar la malla académica; obviamente se requiere de lineamientos teóricos; pero, también hay que fomentar trabajo de campo, sobre todo el que está enfocado a la investigación". Desde hace un año atrás presenta la misma limitación de los otros medios para la contratación de pasantes; por eso no tienen convenios universitarios. Recuerda que la última vez contó con las ideas innovadoras de tres jóvenes, dos pertenecían a una institución privada y, el otro, a una pública; sin embargo, los tres tenían como denominador común una insuficiente ortografía, el desconocimiento de géneros periodísticos, limitadas herramientas para dirigirse al público y un escasez de técnicas de entrevistas. Estos vacíos son producto de la falta de práctica que brindan las universidades y porque son pocos los docentes que enseñan a resolver problemas reales, propios de la profesión.

Con el nacimiento de la Escuela Nueva o Activa (finales del siglo XIX), los conocimientos se vuelven más prácticos que teóricos, lo cual genera la crítica de la escuela tradicional y se asumen nuevos roles: un estudiante activo y participativo, capaz de entender el concepto de utilidad en cada uno de los saberes y que proyecta una visión humanista. Es decir, se propone que la institución se convierta en una comunidad en miniatura, tal como califica Teresa Sanz y Ada Rodríguez del Centro de Estudios para el Perfeccionamiento de la Educación Superior. Ellas, basándose en los conceptos filosóficos de John Dewey, proponen que "la escuela como institución social, debe concentrar los medios que contribuyen a que el estudiante aproveche los recursos que trae al nacer así como para que utilice sus capacidades con fines sociales" (Cepes, 2000).

Con esta concepción nace el modelo "aprender haciendo", configurando el carácter activo- participativo de la formación periodística, el mismo que involucra el planteamiento de problemas auténticos para estimular el pensamiento, las observaciones, las situaciones analíticas 
y las oportunidades para descubrir soluciones. De esta manera nace el Club de Periodismo de Diario El Telégrafo, una iniciativa que consolida la redacción como el eje principal del aprendizaje periodístico y la mejor manera de aprender a escribir es promoviendo la práctica constante como medio de conocer, analizar, valorar y criticar.

\section{La experiencia del Club de Periodismo}

En febrero del 2014, los estudiantes de comunicación de todas las universidades de Guayaquil empezaron a escribir en las páginas del diario, como una manera de fomentar su práctica profesional. Como antecedente de esta iniciativa, un año antes, en Bélgica, se realizó el Tercer Congreso Mundial de Periodismo y Educación y en él se propuso cinco nuevos enfoques para la enseñanza (Morales, 2013): uno de ellos fue convertirlos en empleados de medios de comunicación, técnica pedagógica que consiste en tratar al alumno como empleado de un medio de comunicación para hacerlos sentir inmersos en este rol porque a través del

juego, las personas adquieren, modifican y desarrollan determinadas actitudes y habilidades con mayor facilidad, debido a que disminuye la natural resistencia al cambio y la implicación emocional que se experimente.

Con esta nueva concepción en la enseñanza del periodismo, diario El Telégrafo descubrió estudiantes de colegio, cuya vocación se ha pulido gracias a la práctica precoz en temas de redacción y esto ha mejorado la calidad de estudiantes, al pasar a la universidad. Se trata de una iniciativa que consolida los conocimientos teóricos y los lleva a la práctica a través de la puesta de la redacción de los distintos géneros periodísticos: noticia, entrevista, crónica y reportaje.

Ma. de Lourdes Bermúdez Morán, Jefe de Comunicación Social Subsecretaría de Educación del Distrito de Guayaquil, asevera que este proyecto abarcó dos aristas: el Club de Periodismo como actividad extraescolar y que se ejecutó en los colegios Amarilis Fuentes y la Unidad del Milenio Alfredo Vera Vera. La otra opción fue la participación estudiantil con chicos de segundo de bachillerato e incluyó la opción de Periodismo Comunitario y en el que participaron los colegios Aguirre Abad emblemático D5, Vicente Rocafuerte, Martha Bucaram, Prov. de Imbabura y Prov. de Cañar.

Una vez al mes se capacitaba a los docentes y estudiantes por parte de profesionales de El Telégrafo. Ambos proyectos beneficiaron a los estudiantes porque les dio la oportunidad de ocupar su tiempo libre a través del ocio recreativo obteniendo resultados positivos, quienes pudieron demostrar su capacidad de investigación, siendo una oportunidad para trasmitir sus preocupaciones, presentar temas de interés de los jóvenes en la actualidad y compartir experiencias a través de los reportajes que fueron publicados en el diario El Telégrafo, motivando a muchos a la elección de la carrera de Periodismo o carreras afines a la Comunicación (Bermúdez, 2016)

Los estudiantes y docentes responsables del tema desarrollaron capacidades en liderazgo, trabajo en equipo, seguridad, desenvolvimiento escénico, creatividad para realizar entrevistas impulsando su interés por la investigación y la lectura mejorando su redacción y reportajes. Adicionalmente aprendieron a identificar y diferenciar crónicas, reportajes, noticias, etc. de la mano de los profesionales en la rama. Además, los estudiantes tuvieron la oportunidad de visitar 
las instalaciones del diario para conocer la planta rotativa de impresión y conocer las etapas de pre prensa y post prensa como procesos que suceden antes de la impresión.

Este proyecto fortaleció la relación entre los estudiantes y la comunidad, se fomentó el buen uso del tiempo libre y se estimuló la investigación. Después de dos años de su ejecución, los estudiantes han fortalecido el dominio de la redacción periodística y, por ende, se consolida el mejor desempeño en sus carreras. A la vez, el medio de comunicación se nutre de las experiencias juveniles y su visión en el tratamiento de la información. "Sentíamos que los profesionales que se incorporaban al medio y que estaban recién graduados o en ese proceso, no tenían la preparación suficiente para redactar utilizando fuentes pertinentes, o abordar temas específicos que requeríamos; así que como diario público buscamos integrarlos a la profesión y que sientan que contribuimos con el rol educativo que también tienen los medios de comunicación con toda la colectividad”, indica Orlando Pérez, Director del Diario.

Aunque El Telégrafo establece lineamientos profesionales específicos para abordar los temas, Pérez reconoce que no siempre se acepta todo lo escrito porque se busca calidad de textos y buenos temas; por eso, entiende que la Academia, a través de sus propios coordinadores de carrera, debe apoyar en la asesoría a los estudiantes para la elaboración de sus textos, pues se asumen que ellos sí han tenido experiencia en medios y tendrán la visión y las herramientas para comprender, de mejor manera, lo que quiere el diario.

Gabriela Samaniego, estudiante de la carrera de Periodismo de la Uide, empezó el aprendizaje de la profesión siendo parte del club a nivel de colegio. Al cumplir un año dentro del Club, la evolución es evidente porque está en tercer semestre de la carrera y ha dominado la técnica de la escritura. Ha realizado entrevistas, reportajes y esto le ha permitido vencer los miedos, superar las propias expectativas y ponerse en contacto con personajes públicos para abordar en profundidad un tema específico. Estilos de redacción, composición y demás materias son las que le han permitido ir conociendo, muy de cerca, los estilos de escritura, cuidando ortografía, detalles y, ahora, con enfoque periodístico, manejo de fuentes e investigación.

A pesar de la importancia que tiene la escritura en los estudiantes, no todos consiguen desarrollar las habilidades necesarias para mantenerla. Tener una buena redacción equivale a dar validez al texto, ejercer una estructura ordenada que evidencie el enfoque cognitivo del tema que se aborda; es identificar la comunidad discursiva, promover la reflexión y profundizar el conocimiento. Cada sistema educativo promoverá la adquisición de esta disciplina a través de un aprendizaje verdaderamente integral.

La redacción es la base de la comunicación y la esencia del periodismo. En prensa escrita, escribir bien es un rol inherente del profesional para transmitir el mensaje. La redacción periodística, concebida como orden, desemboca necesariamente en la determinación de contenidos. Por eso se necesita tener una práctica constante en el medio

Para Jefferson Merchán Tomalá, estudiante de la Facultad de Comunicación Social de la Universidad de Guayaquil, debatir ideas con los estudiantes de otras universidades en cada reunión de trabajo, ha sido gratificante para aprender a enfocar los temas. 
Practicar de manera continua permite consultar con catedráticos, leer los trabajos de colegas, visitar páginas web especializadas en redacción; es decir, una infinidad de factores que favorecen el crecimiento en todos los sentidos y eso repercute en la calidad de los trabajos que envío al Telégrafo como en las materias que recibidas en la FACSO. "Personalmente, me siento satisfecho porque no solo en la redacción he visto mejoras sino en la relación con los docentes, el aporte que puedo dar a compañeros y entablar un dialogo de varios temas con colegas y amigos. Es decir un desarrollo integral".

Todo comunicador debe empezar su carrera teniendo como base la redacción porque esta va a estar presente en todos los aspectos de la carrera. No hay mejor satisfacción que el poder escribir correctamente un texto, contrastar fuentes, utilizar los términos adecuados, leer para nutrirse de otras opiniones y esto va a repercutir en nuestra manera de expresarnos y en el desenvolvimiento profesional.

Iván Duarte, estudiante de la Universidad Técnica de Babahoyo, está convencido de que formar parte del Club de Periodismo de diario El Telégrafo lo ha beneficiado mucho porque fomenta su capacidad para investigar. "En el ámbito académico me ha servido para no quedarme solamente con lo que en el aula he aprendido, sino ir más allá, porque los tiempos cambian y la enseñanza es diferente y sé que debo combinar el aprendizaje universitario con el que adquiero en la calle al hacer una nota periodística”.

Independientemente de que existen otras áreas del periodismo como radio y televisión; la redacción es la principal herramienta de la carrera. Y es ésta que debe reforzarse en las aulas de clases y en los trabajos prácticos.

Diego Cuenca Abril, Universidad Católica de Santiago de Guayaquil, reconoce que el Club de Periodismo es una experiencia profesional que cumple sus necesidades como estudiante de Comunicación Social y Periodismo, porque le permitió enfrentarse a la realidad del laboral periodístico y ejercer la profesión con una perspectiva juvenil y con ansias de involucrarse en las historias de la sociedad. "El beneficio que nos da El Club de Periodismo es fortalecer nuestras habilidades que hemos adquirido en el transcurso de los años en nuestra universidad".

La redacción dentro de esta profesión es indispensable y mucho mejor si lo que se escribe está bien estructurado. Tener una redacción impecable es parte del día a día, es una habilidad y un compromiso que se adquiere como parte del rol periodístico.

La mejor historia del mundo se lucirá con una redacción impecable y solo así adquiere el valor exacto y el impacto entre los lectores o audiencia. La clave del periodismo o la matriz productiva del oficio es escribir bien. Si un joven decide inclinarse por las Relaciones Públicas, necesitará redactar boletines o escribir informes; si opta por los medios audiovisuales, también requerirá escribir antes de locutar la noticia; entonces, la clave es escribir bien y tener conocimiento del mundo contemporáneo, dos características que la universidad debe considerar para formar un profesional integral.

\section{Conclusiones}


La enseñanza del periodismo está integrada por diferentes enfoques académicos y, por pertenecer al área social, ha consolidado sus formas y prácticas basadas en los cambios contemporáneos. Esto ha llevado a la formación de sistemas cognitivos de aprendizaje que vinculan la práctica como medio pertinente de resolver problemas de la profesión.

El Club de Periodismo ha fortalecido los valores de sus integrantes y ha destacado el manejo de herramientas prácticas relacionadas con criterios de calidad y prácticas de la profesión. Además, ha fomentado el dominio de aspectos sociales, culturales, políticos, económicos y éticos del ejercicio profesional.

La práctica se ha centrado en el conocimiento de las dificultades del periodismo para resolver las problemáticas existentes. Así mismo favorece el debate democrático, el consumo de noticias y hechos de interés informativos necesarios para realizar investigaciones y mejorar la escritura como medio para ilustrar, corregir y producir textos idóneos.

El Club de Periodismo promueve el aprendizaje significativo pero basado en la práctica real y ante ello las universidades deben replantear su rol y asumir desafíos que conlleva la formación de periodistas reales; es decir prepararlos para el desarrollo integral de su profesión.

\section{Bibliografía}

Azofra, V., \& Femández, A. (1992). Evolución reciente de la modema teoría financiera. Anales de Ciencias Económicas y Empresariales , NQ7, 111-126.

Acosta Damas, M. (2009). Tesis de Doctorado. La Entrevista En El Sistema Informativo De La Televisión Cubana: retos en la sociedad contemporánea. La Habana, La Habana, Cuba: Universidad de La Habana. Facultad de Comunicación Social.

Adelaida García M. (s.f). escribir la verdad. argumento de autoridad, guayaquil.

Almenara, J. C. (15 de Junio de 2002). La introducción del vídeo como instrumento de conocimiento en la enseñanza universitaria. Sevilla, Sevilla, España. Retrieved 10 de Agosto de 2015 from http://tecnologiaedu.us.es/revistaslibros/Artg-ice2.html

Alonso, E. G., Ocegueda, V., \& Castro, E. (2006). Teoria de las organizaciones. Jalisco: Umbral.

Andrade, H. (2005). Comunicación organizacional interna. Eapaña: Gesbiblo, S. L.

Aparicio, A. (2011). Retrieved 23 de Agosto de 2015 from https://www.uam.es/personal_pdi/stmaria/jmurillo/Met_Inves_Avan/Presentaciones/Cues tionario_\%28trab\%29.pdf

Arrieta, B. (2011). Comprension lectora y redaccion en estudiantes Universitarios. Retrieved 27 de Enero de 2016 from http://www.rieoei.org/deloslectores/825Barrieta.PDF

Bernal, C. A. (2010). Metodología de la investigación. Bogota: Pearson. 
Bermúdez, M. L. (2016). Jefe de Comunicación Social Subsecretaría de Educación del Distrito de Guayaquil. (G. Samaniego, Interviewer)

Blank, L., \& Tarquin, A. (2002). Ingeniería Económica. México D.F.: McGraw Hill.

Cabrero, J. (1989). Tecnología educativa: utilización didáctica del video. Barcelona.: PPU.

Calvo, E. (Junio de 2011). Información audiovisual, multimedia y educación. 10 . Granada., España.: Etc@net. Retrieved 22 de Septiembre de 2015 from http://www.ugr.es/ sevimeco/revistaeticanet/index.htm

Canet., F. (2009). Narrativa audiovisual: estrategias y recursos. Madrid: Sìntesis.

Camba, J. (2013). Maneras de ser periodista . Madrid: Principes de Vergara.

Carvajal, G. (2014). Desafíos Periodisticos . Focus, ESPOL , 4,5.

Catarina. (2014). El diseño en conjunto con otras diciplinas. Guayaquil: Catarina.udlap.

Cepes. (2000). Tendencias Pedagógicas en la realidad educativa actual. Bolivia: Editorial Universitaria.

Charles Duncombe. (2014). Gramatica en publicidad. informativo, Guayaquil.

Clarin, E. (18 de Marzo de 2014). Afirman que el sms no afecta la ortografía de los adolescente.

Cnnexpansion. (6 de octubre de 2011). From La mitad de los profesionistas no ejerce: http://www.cnnexpansion.com/mi-carrera/2011/10/05/la-mitad-de-los-profesionistas-noejerce

Court Monteverde, E. (2012). Finanzas Corporativas. Buenos Aires: Cengage Learning.

Cobo, M. d. (2016 de Enero de 2016). ¿Para qué sirve la RAE? El Telégrafo, Cartón Piedra, p. 28.

Ezquerra, Á. (2010). Desarrollo audiovisual de contenidos científico-educativos. Enseñanza de las ciencias. , 28 (3), 353-366.

El Universo. (30 de Octubre de 2015). Retrieved 3 de Febrero de 2016 from Jovenes y las redes sociales: http://www.eluniverso.com/vida-estilo/2015/10/03/nota/5162600/jovenes-usoredes-sociales-hacen-crecer-acceso-internet

El Mundo es. (22 de julio de 2009). ElMundoes. From Los periódicos marcan todavía la agenda política': http://www.elmundo.es/elmundo/2009/07/22/comunicacion/1248250050.html

David Ogilvy. (1990). publicidad. Guayaquil: pinterest. 
Damodaran, A. (2005). Valuation Approaches and Metrics: A Survey of the Theory. Hanover: Foundations and Trends in Finance.

Damodaran, A. (2001). The Dark Side of Valuation. Prentice Hall.

Diario El País. (29 de octubre de 2014). ElPaís.es. From Las carreras con mayor tasa de empleo: http://elpais.com/elpais/2014/10/28/media/1414529398_550532.html

Fernández, M. C. (1997). Influencia del montaje en el lenguaje audiovisual. Madrid.: Libertarias.

Fernández, P. (1998). Valoración de Empresas. Barcelona: Gestión 200.

Fernández, P. (noviembre de 2008). IESE Business School. Retrieved 30 de agosto de 2013 from Universidad de Navarra: http://ssrn.com/abstract=1266623

Fernández, P. (2008). Métodos de Valoración de Empresas. CIIF , 52.

Franco, G. (2008). Blog del Medio. From ¿Cómo escribir para la web?: http://blogdelmedio.com/libros/como-escribir-para-la-web-guillermo-franco/

Gallegos Muñoz, C., \& Medina Giacomozzi, A. (2011). Determinación del valor económico añadido: un modelo alternativo. Contabilidad y Negocios , 18.

Garcia, M. A. (s.f.). Academia. Retrieved 11 de Febrero de 2016 from Relaciones Públicas $\begin{array}{llll}\text { Estrategias } & \mathrm{y} & \text { Tácticas de Comunicación Integradora: }\end{array}$ Http://Www.Academia.Edu/7823071/Relaciones_P\%C3\%9ablicas_Estrategias_Y_T\%C3 \%A1cticas_De_Comunicaci\%C3\%93N_Integradora

García-Abadillo, C. (2009). elmundoes. $\quad$ From http://www.elmundo.es/elmundo/2009/07/22/comunicacion/1248250050.html

Gómez, M. (2007). La comunicación en las organizaciones para la mejora de productividad.

Hernández, J. (2004). Periodismo ¿Oficio imposible? Quito: Quipus.

Hidalgo, A. L. (2013). Periodismo de inmersión . España: Comunicación Social .

Jurado, R. (22 de enero de 2015). Ley de Comunicación. (G. Samaniego, Interviewer)

José Martín. (s.f.). escritura. argumento de autoridad, guayaquil.

Jordán , R., \& Panchana, A. (2011). The handbook of Spanish Languaje Media. From Los medios de comunicación en Ecuador: https://rodrigojordan.files.wordpress.com/2010/05/los-mcsen-ecuador.pdf

Jordán, R., \& Panchana, A. Los Medios De Comunicación En Ecuador. Guayaquil. 
Kraftchenko, Oksana; Hernández, Herminia. (2000). Tendencias Pedagógicas en la Realidad Educativa Actual. Bolivia: Editorial Universitaria.

Lizarzaburo, G. (2015). Editor diario Expreso. (A. Intriago, Interviewer)

López Lubian, F., \& De Luna Butz, W. (2002). Finanzas Corporativas en la práctica. Madrid : McGraw Hill.

López, G. (Noviembre de 2004). Una mirada integral que permita descubrir el sentido y respuestas de la universidad en el futuro (2005-2015). From Unesco: http://unesdoc.unesco.org/images/0014/001473/147349so.pdf

North, R. (2006). Frases sobre la escritura y creacion en general.

Murciano, M. (2055). La enseñanza del periodismo, nuevos desafios internos y externos. From http://www.apmadrid.es/images/stories/10.pdf

Margarite Duras. (s.f.). Desafios de la escritura multimedia. Guayaquil: books.

Marketing Directo. (16 de Octubre de 2011). Retrieved 11 de Febrero de 2016 from 20 cosas que todo profesional de las relaciones públicas debería saber hacer: http://www.marketingdirecto.com/actualidad/checklists/20-cosas-que-todo-profesionalde-las-relaciones-publicas-deberia-saber-hacer/

Marr, B., Roos, G., Neely, A., Pike, S., \& Gupta, O. (2004). Hacia la tercera generación en la medición de resultados. Revista de Contabilidad y Dirección , 1, 31-46.

Martínez, T. (s.f). LaNación.com. From El periodismo vuelve a contar historias: http://www.lanacion.com.ar/215253-el-periodismo-vuelve-a-contar-historias

Medrano Samaniego, C. (2006). El poder educativo de la televisión. Revista de psicodidáctica, $11(1), 93-108$.

Michel, G. (1990). Para leer a los medios, prensa, radio,cine y Tv. México: Trillas.

Milla Gutiérrez, A., \& Martínez Pedrós, D. (n.d.). Altair Consultores. Retrieved Agosto de 2013 from http://www.altairconsultores.com/images/stories/publicaciones/Libro_Valoracion.pdf

Modigliani, F. y. (1958). The Cost of Capital, Corporation Finance and the Theory. American Economic Review , 261-297.

Monje, S. (2011). Revista latina de Comunicación Social. Retrieved 3 de Febrero de 2016 from http://www.redalyc.org/html/819/81921340020/index.html

Morales, F. (2013). Montaje audiovisual: teoría, técnica y métodos de control. UOC.

Olaizola, A. (2005). El ensayo como herramienta de enseñanza . Calí: Comunicaciones. 
Punin, I. (julio de 2012). Razón y Palabra. From Los Estudios de Comunicación SocialPeriodismo en el Ecuador, una visión crítica al rol de la Universidad y La Academia: http://www.razonypalabra.org.mx/N/N79/V79/66_Punin_V79.pdf

Pacheco, R. (2005). Competencias claves para la comunicacion organizacional. Revista Latinoamericana de Comunicacion Chasqui , 72.

Palenzuela, V. A., \& Herrero, G. d. (2008). La huella indeleble de Modigliani y Miller: MM. Boletín de estudios económicos 63 , 373-401.

Pandit, N. (Diciembre de 1996). "La Creación de la teoría: una aplicación reciente del método puesto a tierra de la teoría”. Retrieved 3 de Agosto de 2015 from http://www.nova.edu

Pereyra, T. M. (2008). Valoración de empresas: Una revisión de métodos actuales. Documento de trabajo No. 41, Facultad Administración y Ciencias Sociales, Universidad ORT Uruguay , 34.

Perona., A. M. (2010). Ensayo sobre video, documental y cine. Còrdova: Brujas.

Portal, R., \& otros, y. (2008). Comunicación para el desarrollo: selección de lecturas. La Habana, La Habana, Cuba: Editorial Félix Varela.

Prieto Castillo, D. (2006). El interaprendizaje como clave de la educomunicación en mediaciones. Universidad Minuto de Dios (6).

Saladrigas Medina, H. (2005). Comunicación organizacional: Matrices teóricas y enfoques comunicativos. Revista Latina de Comunicación Social .

Sánchez, S. (1996). La comunicación positiva y el entorno organizaional.

Sancho, J., Vilches, A., \& Gil, D. (2010). Los documentales cientìficos como instrumentos de educaciòn para la sostenibilidad. Eureka, 7 (3), 667-681.

Sandoval, T. (1998). Entrevista a "Mariano Cebrian comenta los últimos y los próximos cambios en comunicación audiovisual”. Revista Latina de Comunicación Social. (7).

SECOM. (12 de Agosto de 2015). Ley de Comunicación . El Telégrafo , pp. 3-4.

Stewart, G. (2000). En busca del valor. Barcelona: Gestión.

RAE. (2010). Retrieved 2 de Febrero de 2016 from http://dle.rae.es/?id=RG9EvWw

Ramos, J. L. (2002). ¿Qué es el vídeo educativo? Madrid, Madrid, España.

Romero, L. Á. (2010). Principios de contabilidad. McGraw Hill.

Tamara Aguilar B. (2016). La factoria. Entevista publicitaria, (p. 1). Guayaquil. 
Tipos de obras audiovisuales. (Octubre de 2015). Cine y fotografía .

Tirira, M. (2013). La escritura y las redes sociales. Retrieved 3 de Febrero de 2016 from https://www.usfq.edu.ec/publicaciones/para_el_aula/Documents/para_el_aula_08/pea_00 8_0010.pdf

Tostado, V. (1999). Manual de producción de video: un enfoque integral. Alhambra.

Unesco. (2007). From http://unesdoc.unesco.org/images/0015/001512/151209s.pdf

Unesco. (9 de Octubre de 1998). Declaracion Mundial Sobre La Educacion Superior. From Conferencia Mundial Sobre La Educacion Superior: http://www.unesco.org/education/educprog/wche/declaration_spa.htm

Unesco. (1998). Transdisciplinarity stimulating synergies, integrating knowledge, Unesco 1998 Transdisciplinarity stimulating synergies - División of Philosophy and Ethics .

Universia. (30 de abril de 2013). UniversiaChile. From http://noticias.universia.cl/enportada/noticia/2013/04/30/1020418/revisa-9-profesiones-estado-saturacion.html

Universidad Catolica de Pereira. (2010). impacto de las practicas profesionales en comunicacion organizacional.

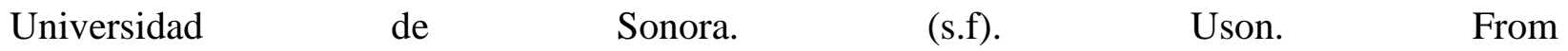
http://tesis.uson.mx/digital/tesis/docs/7564/Capitulo2.pdf

Vanegas, H. (2014). los estudiantes no saben escribir. Retrieved 3 de Febrero de 2016 from http://repository.ut.edu.co/bitstream/001/1166/1/RIUT-BHA-spa-2014-

La\%20escritura\%20y\%20las\%20redes\%20sociales.pdf

Villalobos, f. (S/F). Centro Internacional de Estudios Superiores de Comunicación para America Latina . From http://www.monografias.com/trabajos908/formacion-periodistas/formacionperiodistas2.shtml

Wigodski, J. (14 de Julio de 2010). Metodología del investigación. Retrieved 19 de Julio de 2015 from http://metodologiaeninvestigacion.blogspot.com/2010/07/poblacion-y-muestra.html

Zurita, P. (2014). Jefa del Dpto. Recurso Humanos de Diario El Universo. (C. Drouet, Interviewer) 